\title{
A MORE GENERALIZED GRONWALL-LIKE INTEGRAL INEQUALITY WITH APPLICATIONS
}

\author{
QINGHUA MA and LOKENATH DEBNATH
}

\author{
Received 5 May 2002
}

\begin{abstract}
This paper deals with a new Gronwall-like integral inequality which is a generalization of integral inequalities proved by Engler (1989) and Pachpatte (1992). The new Gronwall-like integral inequality can be used in various problems in the theory of certain class of ordinary and integral equations.
\end{abstract}

2000 Mathematics Subject Classification: 26D10, 26D15.

1. Introduction. It is well known that integral inequalities play a very crucial role in the study of differential equations, integral equations, functionaldifferential equations, and integro-differential equations. Besides the famous Gronwall-Bellman inequality and its first nonlinear generalization by Bihari (see Bellman and Cooke [1]), there are several other very useful Gronwall-like inequalities. Haraux [3, Corollary 16, page 139] derived one Gronwall-like inequality and used it to prove the existence of solutions of wave equations with logarithmic nonlinearities. On the other hand, Engler [2] utilized the following slight variant of inequality due to Haraux [3, page 139] in the study of global regular solutions for the dynamic antiplane shear problem in nonlinear viscoelasticity.

LEMMA 1.1. Let $c>0$ and $a \in L^{1}\left(I=[0, T], \mathbb{R}_{+}=[0, \infty)\right)$, and assume that the function $w: I \rightarrow[1, \infty)$ satisfies

$$
w(t) \leq c\left(1+\int_{0}^{t} a(s) w(s) \log w(s) d s\right), \quad 0 \leq t \leq T,
$$

then

$$
w(t) \leq c \exp \left(\int_{0}^{t} a(s) d s\right), \quad 0 \leq t \leq T .
$$

Pachpatte [4] obtained one generalization of Lemma 1.1 which can be stated as follows. 
LEMMA 1.2. Let $p_{i} \in L^{1}\left(I, \mathbb{R}_{0}=(0, \infty)\right)$ and $p \in L^{1}\left(I, \mathbb{R}_{+}\right)$. Let $g$ be a continuously differentiable function defined on $\mathbb{R}_{+}$, and $g>0$ and $g^{\prime} \geq 0$ on $\mathbb{R}_{0}$. If $u: I \rightarrow \mathbb{R}_{1}=[1, \infty)$ satisfies

$$
u(t) \leq u_{0}+H\left[t, p_{1}, p_{2}, \ldots, p_{n-1}, p u g(\log u)\right], \quad t \in[0, T],
$$

where $u_{0} \geq 1$ is a constant,

$$
\begin{aligned}
& H\left[t, p_{1}, p_{2}, \ldots, p_{n-1}, p u g(\log u)\right] \\
& \quad=\int_{0}^{t} p_{1}\left(t_{1}\right) \int_{0}^{t_{1}} p_{2}\left(t_{2}\right) \cdots \int_{0}^{t_{n-2}} p_{n-1}\left(t_{n-1}\right) \int_{0}^{t_{n-1}} p u g(\log u) d t_{n} \cdots d t_{2} d t_{1},
\end{aligned}
$$

then, for $0 \leq t \leq t_{1}, t, t_{1} \in[0, T]$,

$$
u(t) \leq \exp G^{-1}\left[G\left(\log u_{0}\right)+H\left[t, p_{1}, p_{2}, \ldots, p_{n-1}, p\right]\right]
$$

where

$$
G(r)=\int_{r_{0}}^{r} \frac{d s}{g(s)}, \quad r_{0}>0, r \geq r_{0}
$$

$G^{-1}$ is the inverse of $G$ and $t_{1}$ is chosen so that

$$
G\left(\log u_{0}\right)+H\left[t, p_{1}, p_{2}, \ldots, p_{n-1}, p\right] \in \operatorname{Dom}\left(G^{-1}\right)
$$

for $t \in\left[0, t_{1}\right], t_{1} \in[0, T]$.

The aim of the present paper is to establish a new generalization of all the inequalities discussed in the above lemmas. One application example is also included.

2. Main results. For convenience, we give some basic notations and definitions which will be used in our subsequent discussion. Let $I=[0, T], T>0$, be finite but can be arbitrarily large. Let $\mathbb{R}=(-\infty, \infty), \mathbb{R}_{+}=[0, \infty), \mathbb{R}_{0}=(0, \infty)$, and $\mathbb{R}_{1}=[1, \infty)$. We denote by $L^{1}(I, \mathbb{R})$ the class of all measurable functions $p(t)$ defined on the set $I$ and with range in the set $\mathbb{R}$ with satisfying $\int_{0}^{T}|p(t)| d t<\infty$, and denote by $C^{k}(M, S)$ the class of all $k$-times continuously differentiable functions on the set $M$ with range in the set $S$. We define the differential operators $L_{i}, 0 \leq i \leq n$, by

$$
L_{0} x(t)=x(t), \quad L_{i} x(t)=\frac{1}{p_{i}(t)} \frac{d}{d t}\left(L_{i-1} x(t)\right), \quad 1 \leq i \leq n,
$$


with $p_{n}(t)=1$, where $x(t)$ and $p_{i}(t)>0$ are some functions defined on $I$. For $t \in I$ and some functions $q_{j}(t)>0, j=1, \ldots, n-1$ and $q(t) \geq 0$ defined on $I$, we define

$$
H\left[t, q_{1}, q_{2}, \ldots, q_{n-1}, q\right]=\int_{0}^{t} q_{1}\left(t_{1}\right) \int_{0}^{t_{1}} q_{2}\left(t_{2}\right) \cdots \int_{0}^{t_{n-1}} q\left(t_{n}\right) d t_{n} d t_{n-1} \cdots d t_{1},
$$

where $t_{0}=t$.

A more generalized version of the inequality appearing in Lemma 1.2 is given in the following theorem.

THEOREM 2.1. Let $p_{i} \in L^{1}\left(I, \mathbb{R}_{0}\right)$ and $p \in L^{1}\left(I, \mathbb{R}_{+}\right)$. Let further $g(t) \in$ $C^{1}\left(\mathbb{R}_{+}, \mathbb{R}_{+}\right)$with satisfying $g>0$ and $g^{\prime} \geq 0$ on $\mathbb{R}_{0}$ and $\varphi \in C^{2}\left(\mathbb{R}_{+}, \mathbb{R}_{+}\right)$with $\varphi^{\prime}(u)>0$ for $u>0$ and nondecreasing. If $u: I \rightarrow \mathbb{R}_{+}$satisfies

$$
\varphi(u(t)) \leq u_{0}+H\left[t, p_{1}, p_{2}, \ldots, p_{n-1}, p \varphi^{\prime}(u) g(u)\right], \quad t \in I,
$$

where $u_{0} \geq 0$ is a constant, then, for $t \in I_{1}=\left[0, t_{1}\right] \subset I$,

$$
u(t) \leq G^{-1}\left[G\left(\varphi^{-1}\left(u_{0}\right)\right)+H\left[t, p_{1}, p_{2}, \ldots, p_{n-1}, p\right]\right], \quad t \in I_{1},
$$

where

$$
G(r)=\int_{r_{0}}^{r} \frac{d s}{g(s)}, \quad r_{0}>0, r \geq r_{0}
$$

and $G^{-1}$ is the inverse function of $G$ and $t_{1}$ is chosen so that

$$
G\left(\varphi^{-1}\left(u_{0}\right)\right)+H\left[t, p_{1}, p_{2}, \ldots, p_{n-1}, p\right] \in \operatorname{Dom}\left(G^{-1}\right)
$$

for $t \in I_{1}$.

Proof. Let $\varepsilon>0$ be an arbitrary small constant and define on $I$ a nondecreasing function

$$
v_{\varepsilon}(t)=u_{0}+\varepsilon+H\left[t, p_{1}, p_{2}, \ldots, p_{n-1}, p \varphi^{\prime}(u) g(u)\right]
$$

From (2.3) and (2.7), we have

$$
\begin{gathered}
u(t) \leq \varphi^{-1}\left(v_{\varepsilon}(t)\right), \quad t \in I, \\
v_{\varepsilon}(0)=u_{0}+\varepsilon>0, \\
L_{n} v_{\varepsilon}(t)=p(t) \varphi^{\prime}(u(t)) g(u(t)) .
\end{gathered}
$$

Since $\varphi^{\prime}$ and $g$ are nondecreasing, by (2.8) and (2.9), we observe that

$$
L_{n} v_{\varepsilon}(t) \leq p(t) \varphi^{\prime}\left[\varphi^{-1}\left(v_{\varepsilon}(t)\right)\right] g\left[\varphi^{-1}\left(v_{\varepsilon}(t)\right)\right]
$$


Using the fact that $v_{\varepsilon}(t)$ is positive,

$$
\frac{d}{d t}\left(\varphi^{\prime}\left[\varphi^{-1}\left(v_{\varepsilon}(t)\right)\right]\right)=\varphi^{\prime \prime}\left[\varphi^{-1}\left(v_{\varepsilon}(t)\right)\right] \times \frac{1}{\varphi^{\prime}\left(v_{\varepsilon}(t)\right)} \times \frac{d v_{\varepsilon}(t)}{d t} \geq 0
$$

and $L_{n} v_{\varepsilon}(t) \geq 0$ for $t \in I$, it follows from (2.10) that

$$
\begin{aligned}
\frac{L_{n} v_{\varepsilon}(t)}{\varphi^{\prime}\left[\varphi^{-1}\left(v_{\varepsilon}(t)\right)\right]} \leq & p(t) g\left[\varphi^{-1}\left(v_{\varepsilon}(t)\right)\right] \\
& +\frac{L_{n-1} v_{\varepsilon}(t) \times(d / d t)\left(\varphi^{\prime}\left[\varphi^{-1}\left(v_{\varepsilon}(t)\right)\right]\right)}{\varphi^{\prime 2}\left[\varphi^{-1}\left(v_{\varepsilon}(t)\right)\right]}
\end{aligned}
$$

that is,

$$
\frac{d}{d t}\left(\frac{L_{n-1} v_{\varepsilon}(t)}{\varphi^{\prime}\left[\varphi^{-1}\left(v_{\varepsilon}(t)\right)\right]}\right) \leq p(t) g\left[\varphi^{-1}\left(v_{\varepsilon}(t)\right)\right]
$$

Integrating (2.13) from 0 to $t$ and using the fact that $L_{n-1} v_{\varepsilon}(0)=0$ give

$$
\frac{L_{n-1} v_{\varepsilon}(t)}{\varphi^{\prime}\left[\varphi^{-1}\left(v_{\varepsilon}(t)\right)\right]} \leq \int_{0}^{t} p\left(t_{n}\right) g\left[\varphi^{-1}\left(v_{\varepsilon}\left(t_{n}\right)\right)\right] d t_{n}
$$

It also follows from (2.14) that

$$
\frac{d}{d t}\left(\frac{L_{n-2} v_{\varepsilon}(t)}{\varphi^{\prime}\left[\varphi^{-1}\left(v_{\varepsilon}(t)\right)\right]}\right) \leq p_{n-1}(t) \int_{0}^{t} p\left(t_{n}\right) g\left[\varphi^{-1}\left(v_{\varepsilon}\left(t_{n}\right)\right)\right] d t_{n}
$$

which, upon integrating from 0 to $t$ and using the fact that $L_{n-2} v_{\varepsilon}(0)=0$, leads to

$$
\frac{L_{n-2} v_{\varepsilon}(t)}{\varphi^{\prime}\left[\varphi^{-1}\left(v_{\varepsilon}(t)\right)\right]} \leq \int_{0}^{t} p_{n-1}\left(t_{n-1}\right) \int_{0}^{t_{n-1}} p\left(t_{n}\right) g\left[\varphi^{-1}\left(v_{\varepsilon}\left(t_{n}\right)\right)\right] d t_{n} d t_{n-1} \text {. }
$$

Repeating the above argument successively, we obtain

$$
\begin{aligned}
\frac{(d / d t) v_{\varepsilon}(t)}{\varphi^{\prime}\left[\varphi^{-1}\left(v_{\varepsilon}(t)\right)\right]} \leq & p_{1}(t) \int_{0}^{t} p_{2}\left(t_{2}\right) \int_{0}^{t_{2}} p_{3}\left(t_{3}\right) \cdots \int_{0}^{t_{n-2}} p_{n-1}\left(t_{n-1}\right) \\
& \cdot \int_{0}^{t_{n-1}} p\left(t_{n}\right) g\left[\varphi^{-1}\left(v_{\varepsilon}\left(t_{n}\right)\right)\right] d t_{n} d t_{n-1} \cdots d t_{3} d t_{2} .
\end{aligned}
$$


For any invertible and continuously differentiable function $a(t)$, by changing the variable $\eta=a^{-1}(\xi)$, we have

$$
\int \frac{d \xi}{a^{\prime}\left[a^{-1}(\xi)\right]}=\int \frac{a^{\prime}(\eta)}{a^{\prime}(\eta)} d \eta=\eta+c=a^{-1}(\xi)+c
$$

Using the above fact and integrating (2.17) from 0 to $t$, we obtain

$$
\varphi^{-1}\left(v_{\varepsilon}(t)\right) \leq \varphi^{-1}\left(u_{0}+\varepsilon\right)+H\left[t, p_{1}, p_{2}, \ldots, p_{n-1}, p g\left[\varphi^{-1}\left(v_{\varepsilon}(t)\right)\right]\right]
$$

Define a function $w(t)$ by

$$
w(t)=\varphi^{-1}\left(u_{0}+\varepsilon\right)+H\left[t, p_{1}, p_{2}, \ldots, p_{n-1}, p g\left[\varphi^{-1}\left(v_{\varepsilon}(t)\right)\right]\right]
$$

then

$$
\varphi^{-1}\left(v_{\varepsilon}(t)\right) \leq w(t), \quad t \in I,
$$

and $w(0)=\varphi^{-1}\left(u_{0}+\varepsilon\right)>0$.

It follows from (2.19) and (2.21) that

$$
L_{n} w(t)=p(t) g\left[\varphi^{-1}\left(v_{\varepsilon}(t)\right)\right] \leq p(t) g(w(t))
$$

From (2.22) and using the fact that $g(w(t))$ is positive, $g^{\prime}(w(t)) \geq 0, L_{n-1} w(t) \geq$ 0 for $t \in I$, we observe that

$$
\frac{L_{n} w(t)}{g(w(t))} \leq p(t)+\frac{[(d / d t) g(w(t))] \cdot L_{n-1} w(t)}{g^{2}(w(t))}
$$

that is,

$$
\frac{d}{d t}\left[\frac{L_{n-1} w(t)}{g(w(t))}\right] \leq p(t)
$$

Starting with (2.24) and using the same steps as used from (2.13) to (2.17), we derive that

$$
\begin{aligned}
\frac{(d / d t) w(t)}{g(w(t))} \leq & p_{1}(t) \int_{0}^{t} p_{2}\left(t_{2}\right) \int_{0}^{t_{2}} p_{3}\left(t_{3}\right) \cdots \int_{0}^{t_{n-2}} p_{n-1}\left(t_{n-1}\right) \\
& \cdot \int_{0}^{t_{n-1}} p\left(t_{n}\right) d t_{n} d t_{n-1} \cdots d t_{3} d t_{2} .
\end{aligned}
$$


Now, by the definition of $G$, keeping $t=t_{1}$ and integrating both sides of (2.25) from 0 to $t$, give

$$
G(w(t)) \leq G(w(0))+H\left[t, p_{1}, p_{2}, \ldots, p_{n-1}, p\right]
$$

that is,

$$
w(t) \leq G^{-1}\left[G\left(\varphi^{-1}\left(u_{0}+\varepsilon\right)\right)+H\left[t, p_{1}, p_{2}, \ldots, p_{n-1}, p\right]\right], \quad t \in I_{1} .
$$

The desired bound in (2.4) now follows from (2.8), (2.21), and the last inequality (2.27), together with the limit $\varepsilon \rightarrow 0$.

COROLLARY 2.2. Suppose that the functions $p, p_{1}, \ldots, p_{n-1}$ and $g$ are defined as in Theorem 2.1, and $u_{0} \geq 1$ and $k>0$ are constants. If $u: I \rightarrow \mathbb{R}_{1}$ satisfies

$$
u^{k}(t) \leq u_{0}+H\left[t, p_{1}, p_{2}, \ldots, p_{n-1}, p u^{k} g(\log u)\right], \quad t \in I,
$$

then, for $t \in I_{2}$,

$$
u(t) \leq \exp G^{-1}\left[G\left(\frac{1}{k} \log u_{0}\right)+H\left[t, p_{1}, p_{2}, \ldots, p_{n-1}, \frac{1}{k} p\right]\right]
$$

where $G$ and $G^{-1}$ are defined as in Theorem 2.1 and $I_{2}=\left[0, t_{2}\right] \subset I, t_{2}$ is chosen so that

$$
G\left(\frac{1}{k} \log u_{0}\right)+H\left[t, p_{1}, p_{2}, \ldots, p_{n-1}, \frac{1}{k} p\right] \in \operatorname{Dom}\left(G^{-1}\right)
$$

Proof. Changing the variable $u=\exp (v)$ in (2.28) leads to

$$
\begin{aligned}
\exp (k v) & \leq u_{0}+H\left[t, p_{1}, p_{2}, \ldots, p_{n-1}, p \exp (k v) g(v)\right] \\
& =u_{0}+H\left[t, p_{1}, p_{2}, \ldots, p_{n-1}, \frac{p}{k}(\exp (k v))^{\prime} g(v)\right]
\end{aligned}
$$

This inequality is a special case of Theorem 2.1 when $\varphi=\exp (k v)$. By Theorem 2.1, we derive from inequality (2.31) that

$$
v \leq G^{-1}\left[G\left(\frac{1}{k} \log u_{0}\right)+H\left[t, p_{1}, p_{2}, \ldots, p_{n-1}, \frac{1}{k} p\right]\right], \quad t \in I_{2} .
$$

This follows inequality (2.29).

REMARK 2.3. When $k=1$ in Corollary 2.2, we derive the assertion of Lemma 1.2 . 
Letting $\varphi=u^{k}$ ( $k>1$ is a constant) in Theorem 2.1 leads to the following corollary.

COROLlary 2.4. Suppose that the functions $u, p, p_{1}, \ldots, p_{n-1}$ and $g$ are defined as in Theorem 2.1 and $k>1$ is a constant, then the inequality

$$
u^{k}(t) \leq u_{0}+H\left[t, p_{1}, p_{2}, \ldots, p_{n-1}, k p u^{k-1} g(u)\right], \quad t \in I,
$$

implies

$$
u(t) \leq G^{-1}\left[G\left(u_{0}^{1 / k}\right)+H\left[t, p_{1}, p_{2}, \ldots, p_{n-1}, k p\right]\right], \quad t \in I_{3},
$$

where $G$ and $G^{-1}$ are defined as in Theorem 2.1 and $I_{3}=\left[0, t_{3}\right] \subset I, t_{3}$ is chosen so that

$$
G\left(u_{0}^{1 / k}\right)+H\left[t, p_{1}, p_{2}, \ldots, p_{n-1}, k p\right] \in \operatorname{Dom}\left(G^{-1}\right)
$$

REMARK 2.5. Setting by $n=1, k=2, g \equiv 1$ in Corollary 2.4, we arrive at Ou-Iang's integral inequality given in [5].

REMARK 2.6. By choosing other suitable special functions to $\varphi$, we get other interesting inequalities which could not be derived from Lemma 1.2.

3. Application. Consider the differential equation

$$
\begin{gathered}
L_{n} x^{k}(t)=p(t) x^{k-1}(t) f(x(t)), \quad t \in I, \\
L_{i} x^{k}(0)=C_{i-1}, \quad i=1,2, \ldots, n,
\end{gathered}
$$

where $k>1$ and $C_{i-1}, 1 \leq i \leq n$ are constants, $p$ and $f \in C(I, \mathbb{R})$, and $L_{n}$ is defined as in Section 2. It is easy to observe that (3.1) is equivalent to the integral equation

$$
x^{k}(t)=b(t)+H\left[t, p_{1}, p_{2}, \ldots, p_{n-1}, p x^{k-1} f(x)\right], \quad t \in I,
$$

where

$$
b(t)=C_{0}+\sum_{i=1}^{n-1} C_{i} H\left[t, p_{1}, \ldots, p_{i}\right] .
$$

If $|b(t)| \leq u_{0}$ and $|f(u)| \leq g(|u|)$, where $u_{0}$ and $g$ are defined as in Theorem 2.1 , then we derive from (3.2) that

$$
|x(t)|^{k} \leq u_{0}+H\left[t, p_{1}, p_{2}, \ldots, p_{n-1},|p||x|^{k-1} g(|x|)\right], \quad t \in I .
$$


An application of Corollary 2.4 to inequality (3.4) yields

$$
|x(t)| \leq G^{-1}\left[G\left(u_{0}^{1 / k}\right)+H\left[t, p_{1}, p_{2}, \ldots, p_{n-1}, \frac{1}{k}|p|\right]\right], \quad t \in I_{3} \cap J(x),
$$

where $J(x)$ denotes the maximal existent interval of $x(t)$.

ACKNOWLEDGMENTS. The first author's research was supported by NSF of Education Bureau of Guangdong Province of China Grant no. 0176. This work was supported by the Faculty Research Council of the University of Texas - Pan American.

\section{REFERENCES}

[1] R. Bellman and K. L. Cooke, Differential-Difference Equations, Academic Press, New York, 1963.

[2] H. Engler, Global regular solutions for the dynamic antiplane shear problem in nonlinear viscoelasticity, Math. Z. 202 (1989), no. 2, 251-259.

[3] A. Haraux, Nonlinear Evolution Equations-Global Behavior of Solutions, Lecture Notes in Mathematics, vol. 841, Springer-Verlag, Berlin, 1981.

[4] B. G. Pachpatte, On Gronwall-like integral inequalities, Indian J. Pure Appl. Math. 23 (1992), no. 2, 131-140.

[5] Ou Yang-Liang, The boundedness of solutions of linear differential equations $y^{\prime \prime}+$ $A(t) y=0$, Advances in Math. 3 (1957), 409-415 (Chinese).

Qinghua Ma: Department of Information Technology, Guangdong University of Foreign Studies, Guangdong 510420, China

E-mail address: gdqhma@21cn.com

Lokenath Debnath: Department of Mathematics, University of Texas - Pan American, Edinburg, TX 78539, USA

E-mail address: debnath1@panam.edu 


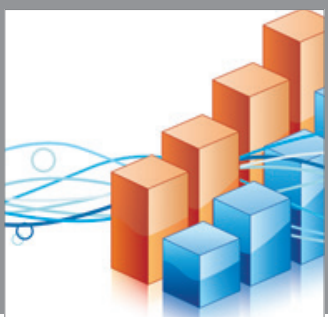

Advances in

Operations Research

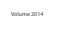

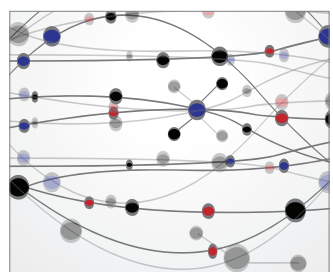

\section{The Scientific} World Journal
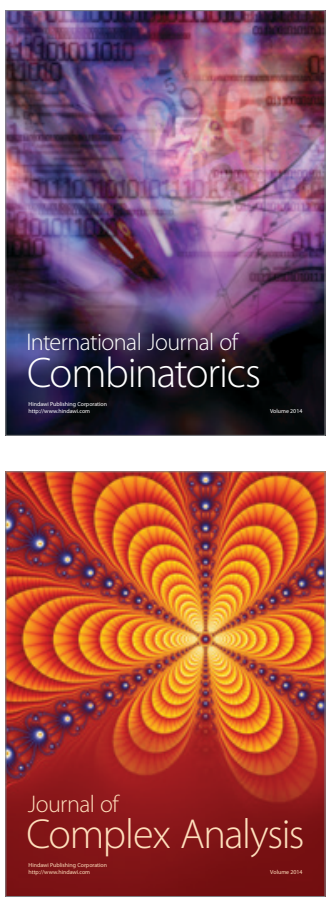

International Journal of

Mathematics and

Mathematical

Sciences
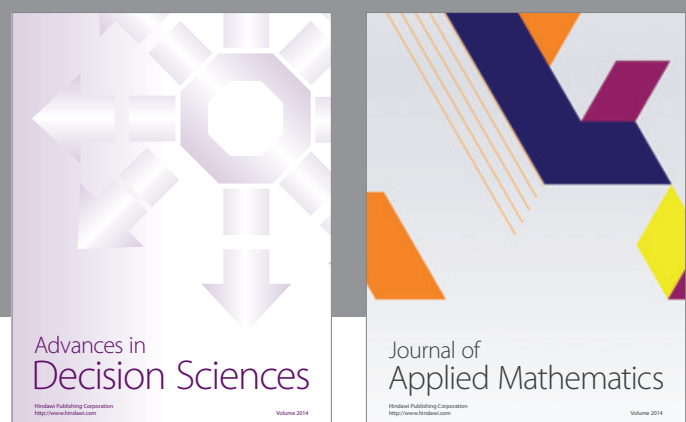

Journal of

Applied Mathematics
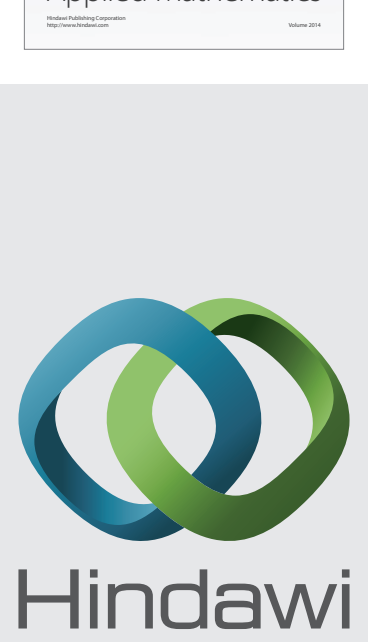

Submit your manuscripts at http://www.hindawi.com
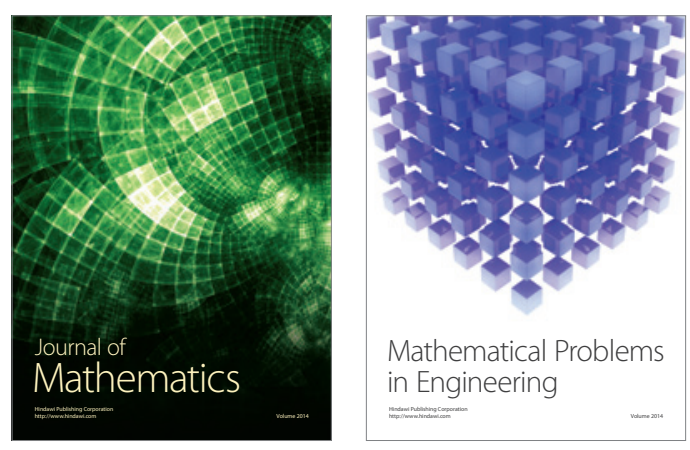

Mathematical Problems in Engineering
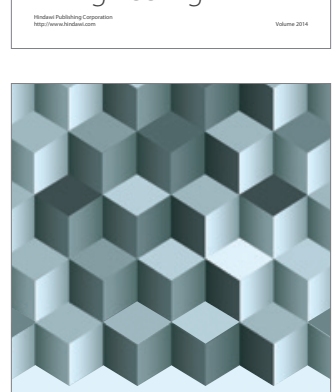

Journal of

Function Spaces
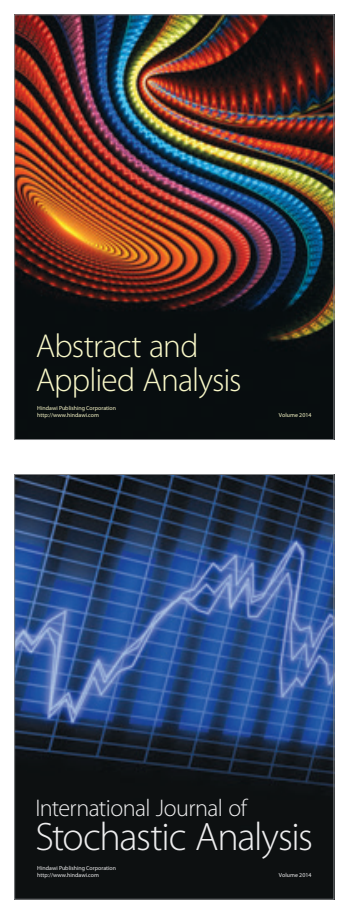

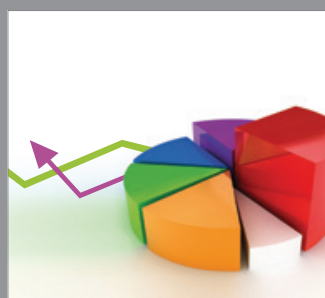

ournal of

Probability and Statistics

Promensencen
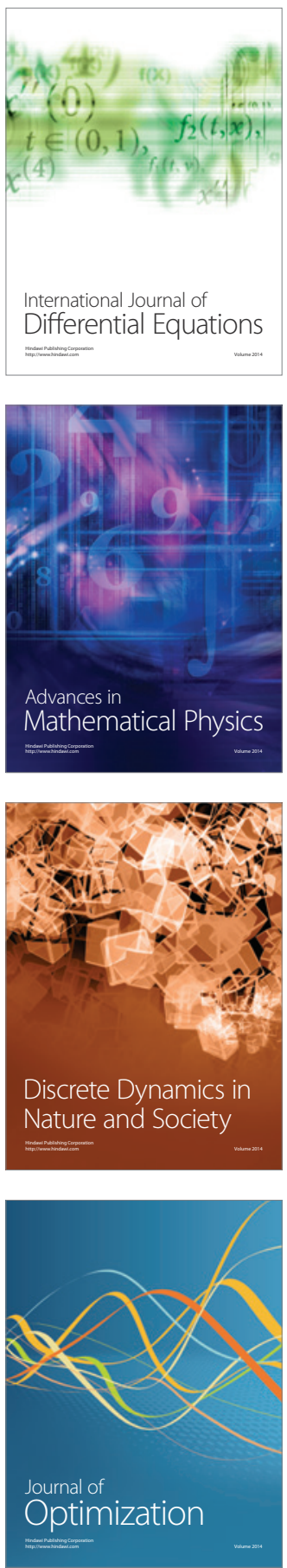